\title{
CRISPR-Cas immunity and mobile DNA: a new superfamily of DNA transposons encoding a Cas1 endonuclease
}

\author{
Alison B Hickman ${ }^{*}$ and Fred Dyda
}

\begin{abstract}
Mobile genetic elements such as DNA transposons are a feature of most genomes. The existence of novel DNA transposons can be inferred when whole genome sequencing reveals the presence of hallmarks of mobile elements such as terminal inverted repeats (TIRs) flanked by target site duplications (TSDs). A recent report describes a new superfamily of DNA transposons in the genomes of a few bacteria and archaea that possess TIRs and TSDs, and encode several conserved genes including a cas 1 endonuclease gene, previously associated only with CRISPR-Cas adaptive immune systems. The data strongly suggests that these elements, designated 'casposons', are likely to be bona fide DNA transposons and that their Cas1 nucleases act as transposases and are possibly still active.
\end{abstract}

Keywords: DNA transposition, V(D)J recombination, CRISPR-Cas, Adaptive immunity, Casposon, Transposable elements, Cas1 nuclease protein

\section{Background}

Mobile genetic elements can modify the genomes of the organisms that harbor them, and their mobility is believed to be an important factor in evolution (reviewed in [1-5]). Mobile elements can affect their host by disrupting genes, modifying control regions, and by introducing new proteins or protein domains into novel genomic locations. One of the best known examples is the RAG1 protein of jawed vertebrates which is a key protein required for the functioning of the adaptive immune system [6], and whose catalytic domain originated from the transposase associated with Transib transposons [7].

One of the most exciting recent advances in microbiology has been the discovery that an adaptive immune system also exists in many bacteria and archaea (reviewed in [8-11]). CRISPR-Cas systems provide a mechanism for prokaryotes to incorporate short stretches of foreign DNA ('spacers') into their genomes to archive sequence information on 'non-self' DNA they have encountered, such as that of viruses or plasmids. This is called the adaptation stage of the immune process. Once integrated, these spacers serve as templates for the synthesis of RNA which

\footnotetext{
* Correspondence: alisonh@helix.nih.gov

Laboratory of Molecular Biology, National Institute of Diabetes and Digestive and Kidney Diseases, National Institutes of Health, Bethesda, MD 20892, USA
}

then directs Cas nucleases to specific foreign nucleic acids in order to degrade them. Several different types of CRISPR systems have been identified, and each is associated with a distinct set of Cas proteins. Only two proteins, Cas1 and Cas2, appear to be strictly conserved among the various CRISPR systems, and they are both metal-dependent nucleases. The structure of the Cas1Cas2 complex from E. coli strain MG1655 has been determined [12].

A recent report by Krupovic et al. [13] presents data suggesting that Cas1 proteins of CRISPR systems originated from a newly identified superfamily of DNA transposons that the authors call 'casposons'. If true, an elegant symmetry emerges in the evolutionary history of the establishment of adaptive immune systems in higher eukaryotes and in bacteria and archaea. Furthermore, the discovery of a novel family of DNA transposases would be a significant addition to the known repertoire of mechanisms by which mobile elements are moved [14].

\section{Main text}

The work of Krupovic et al. builds on a previous report on the evolutionary history of Cas1 proteins which identified two groups of Cas1 proteins not associated with CRISPR loci [9]. One of these groups, designated the 
Cas1-solo group 2, has Cas1 genes in a conserved neighborhood that usually also contains genes for a B family DNA polymerase, an $\mathrm{HNH}$ nuclease, and several helixturn-helix $(\mathrm{HTH})$ domains (Figure 1A). The current analysis reveals that this conserved region is contained between terminal inverted repeats (TIRs) and is flanked by target site duplications (TSDs), hallmarks of DNA transposons encoding RNase $\mathrm{H}$-like transposases (reviewed in $[15,16])$. Krupovic et al. propose that these features suggest that these regions are mobile genetics elements, and that the Cas 1 proteins are required for the integration step of transposition. They further propose that the location of this group of proteins within the Cas1 phylogeny indicates that they likely predate the development of CRISPR-Cas systems.

The parallels between the proposed mechanism of the adaptation step of the CRISPR immune system (reviewed in [17]) and DNA transposition are striking. Cas proteins are responsible for excising a short spacer segment from foreign DNA (typically 32 to 38 bp [11], preceded by a 2 to 5 bp 'protospacer adjacent motif', or PAM) and site-specifically integrating it into a particular genomic location at the leader end of a CRISPR locus. Spacer integration is accompanied by the generation of direct repeats on either side of the spacer that can vary in size from 23 to 55 bp [11]. Thus, if the Cas1 nucleases

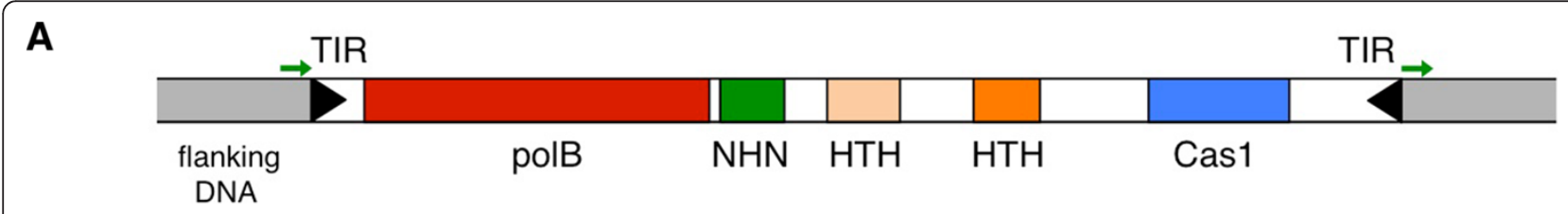

B

TIR-L

TSD

Transposon end (TIR)

MetFor-C1

AAGAAACGTATGCGGGGTAATAGTTATCCCGAAAAAATGCCACTCTTGTAAAACT

MetPsy-C1

ATAAAAAGAAGGTCTAACTCATGACAATAGGAAGAACAATTTCAATTTATCTACC

MetTin-C1

CGCATATTTTTATGGGGATATAGGTAACATCCAAAAATACCACTATTCTAAAAAT

MetMaz-C1

ATAATCTTAATGCGGGGATATAGGTAACTCAAAAAAACGCAACGCATGTGTAAGA

MetMah-C1

CGCACCAACCAATCGGGTTATAGGTAGAATTGAAAAATGCAACCTTAGATAAAAA

MetLum-C1

CGCACTTTTGTGCGGGGTAGTAGTTATCGTACCGATTTGTCACTGTCAAACTATA

AciBoo-C1

CCCCACTACGAGGAGGGGGATATATATACATCCCCTCTTAAGTTCCCTTTTCACA

MetArv-C1

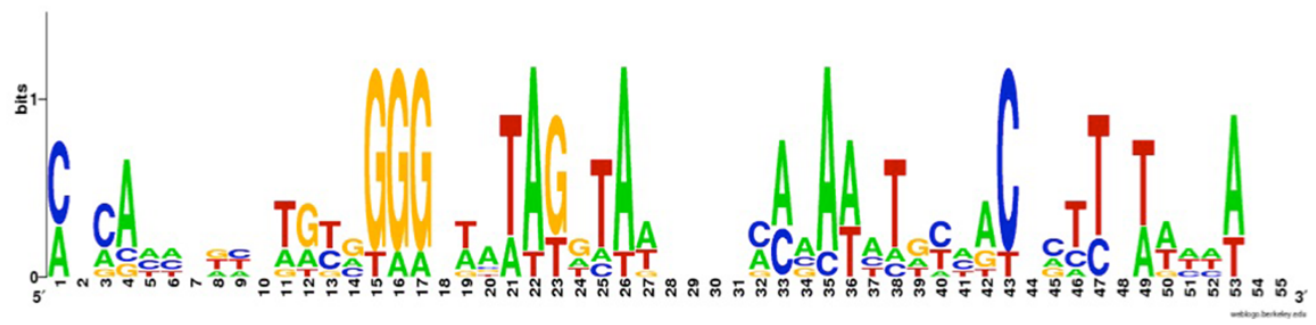

Figure 1 Properties of the family 2 casposons. (A) Predicted common protein-coding genes within family 2 casposons include a PolB family polymerase, an HNH family endonuclease, several HTH domains, and Cas1. The gene color code corresponds to that of Krupovic et al. The green arrows flanking the casposons indicate target site duplications (TSDs). (B) An alignment of the first 41 nucleotides (nt) of casposon family 2 Left End Terminal Inverted Repeats (TIRs) reveals conserved sequence motifs which could be the basis of transposase recognition. Green letters indicate the TSDs and black letters the TIR sequences identified by Krupovic et al., with apparently conserved patterns highlighted in red or blue. Bold black lettering corresponds to nts that were not included in the analysis of Krupovic et al. The aligned sequences and the Accession Number and coordinates for each are: MetFor-C1 [NC_019943;1964105.1964159], MetPsy-C1 [NC_018876;190336.190390], MetTin-C1 [NZ_AZAJ01000001; 3015399..3015453], MetMaz-C1 [NC_003901; 3946587..3946641], MetMah-C1[NC_014002; reverse complement of 1332841..1332895], MetLum-C1 [NZ_CAJE01000015; 159864..159918] AciBoo-C1 [NC_013926; 380309.380363], MetArv-C1 [NC_009464; 2695204.2695258]. 
associated with casposons are involved in catalyzing transposition, they presumably can sequence-specifically recognize their TIRs which for most DNA transposons are longer than $10 \mathrm{bp}[2,15]$. They also appear to exhibit relaxed target DNA recognition properties relative to CRISPR-Cas systems: whereas spacer integration mediated by Cas proteins is site-specific, the genomic locations of casposons suggests that their integration sites are not highly conserved (in line with the integration properties of most RNase H-like DNA transposons with a few notable exceptions, such as the bacterial Tn7 transposon [18]).

One of the main ways that transposon superfamilies are grouped is by the conservation of TIR sequences located at their transposon ends. At first glance, the 19 putative casposon TIR sequences identified and analyzed by Krupovic et al. appear disconcertingly variable both in length and in sequence. However, we find that it is possible to align the TIRs of the sequences corresponding to casposon family 2 members (the most populous casposon family defined in Krupovic et al.) such that a pattern of conserved base pairs emerges within the terminal approximately 20 bp (Figure 1B). This suggests that transposon-specific end recognition by a casposonencoded protein is reasonable. (Casposon families 1 and 3 TIRs can also be aligned to reveal conserved TIR motifs but have fewer representatives than family 2.)

The alignment in Figure 1 also suggests a resolution of a second unusual feature of the sequences presented by Krupovic et al., which is that the TSDs are reported to vary in size from 1 to 27 nucleotides (nt). TSD size is typically highly conserved in Insertion Sequences and DNA transposon superfamilies, rarely varying by more than one or two nt $[15,2]$. This is because TSD size is a direct consequence of the spacing of the staggered cuts generated by a transpososome assembled on target DNA, and it reflects properties of the distinct architecture - in particular the distance between and the orientation of two catalytic sites - of these multimeric protein-DNA complexes. When the TIRs of casposon family 2 are aligned as in Figure 1B, the TSD size (as they are usually defined which does not include any overlap with the TIRs) now converges on $14 \mathrm{bp}$. This is relatively large when compared to TSDs of most characterized transposons, but is substantially less than the range of 23 to $55 \mathrm{nt}$ for the repeat size of CRISPR systems. The thus-aligned TSD sequences also hint at yet another feature of many characterized DNA transposons which is a preferred palindromic target site motif [19].

Finally, it should be noted that all of the casposonassociated Cas1 proteins identified by Krupovic et al. possess the four conserved catalytic residues expected for an active Cas1 nuclease (Supplemental Figure 1 in their report).

\section{Conclusions}

The evidence is compelling that casposons possess some of the expected properties of active DNA transposons. However, as we are only beginning to understand how the multiple Cas proteins in different CRISPR systems mediate immunity, the evolutionary link between the CRISPRassociated Cas1 proteins and the casposon-associated Cas1 proteins provides only limited insight into the possible mechanism of casposon mobility. Many intriguing questions have been raised by the report of Krupovic et al. Since two types of nuclease are often associated with casposons, the Cas 1 proteins and usually an $\mathrm{HNH}$ nuclease, does the latter have a role? If so, do these nucleases work together and interdependently to catalyze excision and integration? How might Cas1 and a B family polymerase collaborate to generate the proposed intermediate of the reaction, an excised transposon flanked by double-strand breaks? How is this related to the transposition mechanism of the superfamily of self-synthesizing Polinton/Mavericks found in eukaryotes $[20,21]$, to which casposons are proposed to be mechanistically related albeit not evolutionarily [13]? Do the recurrent HTH domains identified within casposons (for example, all the Cas1 proteins of casposon family 2 have a conserved HTH appended to their Ctermini) play a role in the recognition of transposon ends or a target site? Clearly, experimental biochemistry is needed to answer these questions.

\section{Abbreviations}

bp: base pair; Cas: CRISPR-associated; CRISPR: Clustered Regularly Interspaced Short Palindromic Repeats; HTH: helix-turn-helix; nt: nucleotides; PAM: protospacer adjacent motif; TIR: terminal inverted repeat; TSD: target site duplication.

\section{Competing interests}

The authors declare that they have no competing interests.

\section{Authors' contributions}

$\mathrm{ABH}$ and FD wrote the text, and approved the final manuscript.

\section{Acknowledgments}

This work was supported by the Intramural Research Program of the NIH, The National Institute of Diabetes and Digestive and Kidney Diseases (NIDDK).

Received: 18 July 2014 Accepted: 11 August 2014

Published: 26 August 2014

\section{References}

1. Deininger PL, Moran JV, Batzer MA, Kazazian HH Jr: Mobile elements and mammalian genome evolution. Curr Opin Genet Dev 2003, 13:651-658.

2. Feschotte $C$, Pritham EJ: DNA transposons and the evolution of eukaryotic genomes. Annu Rev Genet 2007, 41:331-368.

3. Feschotte $C$ : Transposable elements and the evolution of regulatory networks. Nature Rev Genet 2008, 9:397-405.

4. Biémont C: A brief history of the status of transposable elements: from junk DNA to major players in evolution. Genetics 2010, 186:1085-1093.

5. Janicki M, Rooke R, Yang G: Bioinformatics and genomic analysis of transposable elements in eukaryotic genomes. Chromosome Res 2011, 19:787-808

6. Schatz DG, Swanson PC: V(D)J recombination: mechanisms of initiation. Annu Rev Genet 2011, 45:167-202.

7. Kapitonov W, Jurka J: RAG1 core and V(D)J recombination signal sequences were derived from Transib transposons. PLoS Bio/ 2005, 3:e181. 
8. Westra ER, Swarts DC, Staals RHJ, Jore MM, Brouns SJJ, van der Oost J: The CRISPRs, they are a-changin': how prokaryotes generate adaptive immunity. Annu Rev Genet 2012, 46:311-339.

9. Makarova KS, Wolf $\mathrm{YI}$, Koonin EV: The basic building blocks and evolution of CRISPR-Cas systems. Biochem Soc Trans 2013, 41:1392-1400.

10. Sorek R, Lawrence CM, Wiedenheft B: CRISPR-mediated adaptive immune systems in bacteria and archaea. Annu Rev Biochem 2013, 82:237-266.

11. Barrangou R, Marraffini LA: CRISPR-Cas systems: prokaryotes upgrade to adaptive immunity. Mol Cell 2014, 54:234-244.

12. Nuñez JK, Kranzusch PJ, Noeske J, Wright AV, Davies CW, Doudna JA: Cas1Cas2 complex formation mediates spacer acquisition during CRISPR-Cas adaptive immunity. Nature Struct Mol Biol 2014, 21:528-534.

13. Krupovic M, Makarova KS, Forterre P, Prangishvili D, Koonin EV: Casposons: a new superfamily of self-synthesizing DNA transposons at the origin of prokaryotic CRISPR-Cas immunity. BMC Biol 2014, 12:36.

14. Curcio MJ, Derbyshire KM: The outs and ins of transposition: from Mu to kangaroo. Nature Rev Mol Cell Biol 2003, 4:865-877.

15. Chandler M, Mahillon J: Insertion sequences revisited. In Mobile DNA, Volume 2. Edited by Craig NL, Craigie R, Gellert M, Lambowitz A. Washington DC: ASM Press; 2002:305-366.

16. Yuan YW, Wessler SR: The catalytic domain of all eukaryotic cut-and-paste transposase superfamilies. Proc Natl Acad Sci U S A 2011, 108:7884-7889.

17. Fineran PC, Charpentier E: Memory of viral infections by CRISPR-Cas adaptive immune systems: acquisition of new information. Virol 2012, 434:202-209.

18. Peters JE, Craig NL: Tn7: smarter than we thought. Nature Rev Mol Cell Biol 2001, 2:806-814

19. Linheiro RS, Bergman CM: Testing the palindromic target site model for DNA transposon insertion using the Drosophila melanogaster P-element. Nucl Acids Res 2008, 36:6199-6208.

20. Kapitonov W, Jurka J: Self-synthesizing DNA transposons in eukaryotes. Proc Natl Acad Sci U S A 2006, 103:4540-4545.

21. Pritham EJ, Putliwala T, Feschotte C: Mavericks, a novel class of giant transposable elements widespread in eukaryotes and related to DNA viruses. Gene 2007, 390:3-17.

doi:10.1186/1759-8753-5-23

Cite this article as: Hickman and Dyda: CRISPR-Cas immunity and mobile DNA: a new superfamily of DNA transposons encoding a Cas1 endonuclease. Mobile DNA 2014 5:23.

\section{Submit your next manuscript to BioMed Central and take full advantage of:}

- Convenient online submission

- Thorough peer review

- No space constraints or color figure charges

- Immediate publication on acceptance

- Inclusion in PubMed, CAS, Scopus and Google Scholar

- Research which is freely available for redistribution 